\title{
Alice Burrows. L'Alliance française de Buenos Aires de 1914 à 1983, étude des conditions de circulation linguistique.
}

Thèse de doctorat préparée sous la direction de Valérie Spaëth (université Paris Sorbonne Nouvelle) et soutenue le 4 juin 2018 à l'Université Sorbonne Paris Cité, 440 pages.

$\mathrm{PhD}$ thesis prepared under the supervision of Valérie Spaëth (Université Paris Sorbonne Nouvelle) and defended on 4 June 2018 at the Sorbonne University Paris Cité, 440 pages.

Alice Burrows

\section{(2) OpenEdition}

\section{Édition électronique}

URL : https://journals.openedition.org/dhfles/7766

DOI : $10.4000 /$ dhfles. 7766

ISSN : 2221-4038

Éditeur

Société Internationale pour l'Histoire du Français Langue Étrangère ou Seconde

\section{Édition imprimée}

Date de publication : 1 décembre 2020

Pagination : 377-383

ISSN : $0992-7654$

\section{Référence électronique}

Alice Burrows, « Alice Burrows. L'Alliance française de Buenos Aires de 1914 à 1983, étude des conditions de circulation linguistique. ", Documents pour l'histoire du français langue étrangère ou seconde [En ligne], 64-65 | 2020, mis en ligne le 03 février 2021, consulté le 25 mars 2023. URL : http:// journals.openedition.org/dhfles/7766 ; DOl : https://doi.org/10.4000/dhfles.7766 


\section{Alice Burrows. L'Alliance française de Buenos Aires de 1914 à 1983, étude des conditions de circulation linguistique.}

Thèse de doctorat préparée sous la direction de Valérie Spaëth

(université Paris Sorbonne Nouvelle) et soutenue le 4 juin 2018 à

l'Université Sorbonne Paris Cité, 440 pages.

$\mathrm{PhD}$ thesis prepared under the supervision of Valérie Spaëth (Université Paris

Sorbonne Nouvelle) and defended on 4 June 2018 at the Sorbonne University

Paris Cité, 440 pages.

Alice Burrows

\section{Pourquoi étudier l'Alliance française de Buenos Aires de 1914 à 1983 ?}

- Quel est l'objectif de la recherche?

L'objectif de cette thèse est de se saisir des outils de la micro-histoire (Ginzburg 1989), afin d'étudier la circulation des idées linguistiques et didactiques. Ce travail de thèse met en lien la circulation de ces objets intellectuels avec le développement et l'institutionnalisation de la pratique sociale d'enseignement-apprentissage du français. À ce titre, l'Alliance française de Buenos Aires (AFBA) de 1914 à 1983 est comprise comme un lieu prototypique de cette circulation, à l'interface entre la France et l'Argentine et leurs enjeux linguistiques respectifs.

La circulation des idées linguistiques et didactiques, véhiculée par les supports et les individus, entraîne leur traduction en fonction des enjeux des espaces dans lesquels ils circulent. En ce sens, les productions intellectuelles sont interprétées (resémantisées, Espagne 1999). C'est précisément sur cette resémantisation que porte la thèse, 
cherchant donc à savoir comment les enjeux internationaux et locaux influent sur la compréhension et la mise en oeuvre de configurations méthodologiques (Besse 2000). L'étude des circulations linguistiques à l'Alliance française de Buenos Aires de 1914 à 1983 tente donc de comprendre en quoi l'enseignement-apprentissage du français dans cette institution, tout en s'inscrivant parfaitement dans des perspectives nationalistes, en explose les cadres nationaux.

- Pourquoi l'Alliance française?

La création de l'Alliance française à Paris, en 1883, entre en résonnance avec les débats sur la fondation d'une Éducation Nationale sous la III République, tandis que la fondation du Comité à Buenos Aires, en 1893, est marquée par les enjeux communautaires français sur le sol argentin. En effet, l'Alliance française de Buenos Aires (AFBA), se situe à la croisée entre des enjeux argentins pour la diffusion du français et des enjeux français pour cette même diffusion. L'association fondée dix ans après l'Alliance française de Paris par la communauté française de Buenos Aires se veut être un levier pour le maintien des intérêts de la communauté de migration française dans l'espace argentin.

Ainsi, alors que la diffusion du français depuis Paris se fixe pour objectif de conserver intactes les forces vives françaises et de préserver le rapport des Français à la France, la diffusion du français par l'AFBA, depuis Buenos Aires, a pour objectif de structurer la communauté afin, notamment, d'en préserver les intérêts économiques. Dès lors, l'AFBA en contact avec les productions linguistiques et didactiques des espaces français et argentins opère de multiples resémantisations afin de créer des configurations méthodologiques correspondant aux enjeux de préservation communautaire.

- Comment étudier la resémantisation?

L'enseignement-apprentissage au sein de cette institution constitue donc un exemple intéressant pour comprendre comment s'opère la resémantisation des objets intellectuels, sinon dans le champ de la didactique des langues, du moins du FLE. Au demeurant, une étude de la circulation et de l'interface pose le problème de la périodisation et de la domination des espaces dans le découpage temporel (Douki \& Minard 2007). Le choix monographique se double donc d'un découpage non linéaire, fragmenté à parts égales : deux moments de crise internationales européennes qui ont d'importants retentissements en Argentine (1914-1918, 1939-1945), un découpage propre à l'espace européen (l'entre-deux guerres, 1919-1938) et un découpage propre à l'espace argentin (la dernière dictature militaire, 1976-1983). La mise en oeuvre de techniques issues de la micro-histoire, doublée d'une périodisation fragmentée a donc conduit au relevé des traces dans le matériel administratif, politique et pédagogique de ces resémantisations.

\section{L'essor de la « méthode Alliance » : une configuration méthodologique glocale : 1914-1938}

4 La circulation linguistique au cours de la Première Guerre mondiale trouve une expression particulière dans le développement de l'AFBA: la coïncidence entre les intérêts de l'effort de guerre et de l'enseignement-apprentissage du français. La communauté française, prise dans des rapports de lutte économique contre les autres communautés de la capitale (Regalsky 1996), trouve dans l'effort de guerre une expression particulière de la défense de ses intérêts. À ce titre, l'Alliance française de 
Buenos Aires, dont les membres se situent aussi bien dans les groupements d'intérêts économiques que dans les groupements d'intérêts patriotiques, s'impose comme un acteur incontournable de la communauté et impose peu à peu son paradigme constitutif : est ami de la France, celui qui parle (bien) français. Il se met donc en place un lien entre l'institutionnalisation de la pratique sociale d'enseignementapprentissage de la langue, la correction de la langue et un positionnement politique. C'est cette triade qui pousse l'association à questionner l'enseignement-apprentissage du français au-delà des dispositifs, jusque dans les configurations méthodologiques. Le Comité directeur de l'AFBA développe donc, à partir de 1924, des propositions didactiques permettant d'organiser l'enseignement-apprentissage du français au sein de ses cours. Il s'agit de concevoir une «méthode Alliance» permettant de résoudre, selon ses concepteurs, les problèmes d'apprentissage spécifiques aux apprenants du Rio de la Plata. Les manuels sont donc centrés sur une analyse contrastive du français et de l'espagnol. Dans ce cadre, la traduction est utilisée comme un outil au service de la comparaison. L'organisation linguistique du contact entre le français et l'espagnol repose sur une vision de la cohabitation de ces deux langues: l'enseignementapprentissage $\mathrm{du}$ français sert de tuteur réflexif à celui de l'espagnol. Ce positionnement comparatiste soutient le maintien des techniques de traduction et le recours à la grammaire explicite, afin de faire émerger une fonction à la langue française au sein d'un quotidien linguistique hispanophone.

Cette configuration méthodologique posée comme «méthode Alliance » par le Comité directeur de l'AFBA et divisée en plusieurs manuels, échoue dans son entreprise de séduction des politiques linguistiques françaises et argentines. La configuration méthodologique est rejetée par le Ministère qui, en la personne d'Henri Peyre, produit un rapport très négatif des techniques d'enseignement mises au point par l'AFBA. Du côté argentin, les Conseils Nationaux successifs refusent de faire entrer les manuels de la « méthode Alliance » sur la liste officielle des manuels autorisés pour l'enseignement secondaire. Le format de l'enseignement officiel des langues en Argentine repose alors sur de nombreux principes antinomiques à ceux de la "méthode Alliance", directement importés des débats linguistiques européens (exclusion de la traduction, apprentissage par répétition, entre autres traits). Cependant, les choix atypiques de l'AFBA rencontrent un franc succès auprès d'une population scolarisée et universitaire, qui attribue à ces cours des fonctions para-scolaires. L'entre-deux-guerres marque donc à rebours de la reconnaissance politique des propositions didactiques, une période d'essor fulgurante pour la pratique sociale d'enseignement-apprentissage du français, tel qu'il est conçu par l'AFBA.

\section{Déclin de la « méthode Alliance » : une double nationalisation franco-argentine : 1939-1983}

Sous la pression économique du Ministère des Affaires Étrangères, le Comité directeur accepte, contre une augmentation de la subvention, d'intégrer une direction pédagogique directement nommée par Paris. De plus, la communauté française, en perte de vitesse du point de vue migratoire et profondément divisée, quant à un possible ralliement à l'appel du Général de Gaulle, constitue de moins en moins le socle sur lequel s'appuient les actions de l'AFBA, qui se tourne alors pleinement vers la société argentine. Or la montée des fascismes en Europe, trouvent leur pendant dans les 
régimes militaires précédents l'arrivée au pouvoir de Perón, ce qui se traduit très concrètement, dans le système éducatif, par la mise en place de l'éducation au nationalisme (Puiggros 2003). La Seconde Guerre mondiale marque donc une rupture dans les configurations méthodologiques élaborées par l'AFBA pour deux raisons: d'une part, à cause du tournant opéré par ses dispositifs didactiques (augmentation des frais d'inscription, développement d'une offre très avancée de cours de français), d'autre part, en raison de son adhésion aux principes pédagogiques du Conseil National de l'Éducation. Dès lors, les modifications introduites dans le manuel, en particulier les modifications fascisantes concernant les éléments culturels sont particulièrement caractéristiques des phénomènes de circulation. Ainsi, ces modifications sont interprétables dans le cadre du gouvernement de Vichy, mais elles le sont également et surtout dans le cadre de la montée du nationalisme pédagogique argentin, en lien luimême avec la montée des fascismes européens. À ce titre, l'insertion d'éléments culturels en vue de l'éducation au patriotisme reflète aussi bien l'idéologie de Vichy que celle de l'éducation au nationalisme argentin (leçon sur les drapeaux, sur le bon patriote...). En 1939, les enjeux glocaux se situent dans un tout autre cadre que celui de la Première Guerre mondiale: il ne s'agit plus de batailles entre communautés de migration pour la victoire d'une formation mémorielle sur une autre, mais d'une resémantisation des éléments éducatifs fascisants acceptables aussi bien pour le gouvernement argentin que pour le gouvernement français.

7 L'intégration progressive d'une configuration méthodologique alliant enjeux locaux et internationaux débouche sur l'insertion de l'AFBA au sein du champ français de la didactique du français langue étrangère (Porcher 1987). Cette intégration se caractérise par deux faits : d'une part, l'abandon progressif d'une réflexion méthodologique propre et d'autre part, un alignement de l'AFBA avec les objectifs de diffusion linguistique du Ministère des Affaires Étrangères. Le développement de l'AFBA au cours de la dictature est donc le produit d'une situation contradictoire : signant à la fois son apogée et son déclin sur le plan didactique. L'institution, outil du champ français du français langue étrangère se trouve dans une situation de grande intégration sur le territoire argentin, au vu de l'influence de ce champ sur l'enseignement-apprentissage du français en Argentine dans les années 1970 (Varela 2008). Les liens privilégiés entre le CREDIF et l'AFBA en particulier font de l'institution un acteur majeur de la réflexion méthodologique sur l'enseignement-apprentissage du français de 1976 à 1983 à Buenos Aires. Cette situation s'incarne notamment par la création de formations professorales propres à l'AFBA, continues et initiales pour l'enseignement du français. Au demeurant, cette situation entraîne une mise sous tutelle de l'association, dont les configurations méthodologiques deviennent caduques. On constate donc que la rupture opérée par la Seconde Guerre mondiale, conduit paradoxalement l'Alliance française de Buenos Aires à jouer un rôle majeur dans les développements de l'enseignement-apprentissage du français en Argentine, tout en lui ôtant, à terme, ses spécificités méthodologiques. Cette situation se solde par l'adoption d'une réponse universaliste pour l'enseignementapprentissage du français dans laquelle l'AFBA s'engage pleinement à partir de la mise en place d'Archipel. L'adoption d'Archipel marque alors le déclin du rôle central de l'Alliance française dans l'enseignement-apprentissage du français qui se traduit, notamment, par la fermeture de ces formations à destination des enseignants. 


\section{Conclusions et perspectives du travail}

8 La fin d'une réflexion autour des configurations méthodologiques s'incarne dans l'arrêt de la resémantisation des objets didactiques issus du champ français. L'adoption de manuels fabriqués en France depuis les années 1980 a poussé les enseignants, comme les enseignants-chercheurs à se tourner vers d'autres lieux pour l'élaboration méthodologique (universités, instituts...). Il serait essentiel de s'interroger sur le lien entre le déclin de ses resémantisations productives et le déclin général de l'enseignement-apprentissage du français en Argentine, afin de questionner à rebours les effets de la mise en place du champ français du français langue étrangère.

Si cette corrélation reste à faire, le travail d'analyse des resémantisations, opéré par ce travail de thèse, permet de saisir les configurations et les dispositifs didactiques, non comme des objets naturels inscrits dans des contextes, marqués par des cultures, mais comme des produits tendus par des intentions, résultats d'enjeux politiques. Ce constat nous invite à considérer les enjeux glocaux contenus dans les objets didactiques et contribue à éclairer la problématique actuelle du point de vue que la didactique doit adopter, opposant le global au local (Babault, Bento \& Spaëth 2017). Suivant Jean-Loup Amselle (2011), il nous semble primordial d'insister sur le caractère essentiellement hybride des espaces géographiques et de leurs constructions intellectuelles, telles que les configurations didactiques de l'enseignement-apprentissage des langues. Il paraît alors nécessaire de dépasser le débat opposant les partisans de l'universalisme méthodologique aux partisans de la stricte contextualisation en comprenant les configurations méthodologiques et plus largement les objets didactiques comme les résultats scientifiques d'idéologies linguistiques en circulation. Dès lors il importe de comprendre les conditions de ces circulations et les rapports de domination qui y président afin de déterminer le niveau de glocalité des configurations didactiques. Le rapport à la circulation linguistique pourrait ainsi fournir un autre éclairage aux enjeux contenus dans la mise en place des configurations didactiques des langues.

\section{BIBLIOGRAPHIE}

AMSELLE, Jean- Loup (2011). L'Occident décroché : enquête sur les postcolonialismes. Paris : Pluriel. BABAUlt, Sophie, BENTO, Margaret \& SPAËTH, Valérie (2017). Tensions en didactique des langues. Paris : Peter Lang. BESSE, Henri (2000). Propositions pour une typologie des méthodes de langues. Thèse d'État, Université Paris VIII. 3 vol.

ESPAGNE, Michel (1999). Les transferts culturels franco-allemands. Paris : PUF.

DOUKI, Caroline \& MINARD, Philippe (2007). « Histoire globale, histoires connectées : un changement d'échelle historiographique ?». Revue d'Histoire Moderne et Contemporaine, 54-4bis, 7-21. 
GINZBURG, Carlo (1989). Mythes, emblèmes et traces. Morphologie et histoire. Paris : Flammarion. PORCHER, Louis (1987). Champs de signes. Paris : CREDIF.

PUIGGRós, Adriana (2003). Que pasó en la Argentina. Buenos Aires : Galerna.

REGALSKY, Andrés (1996). « Las compañías francesas de ferrocarriles y su repercusión en el desarrollo regional de Santa Fe y Buenos Aires, 1880-1930 ». In Carlos MARICHAL. La banca francesa en América Latina, 1870-1930. México : El Colegio de México. 193-213.

VARELA, Lia (2006). La politique linguistique extérieure de la France et ses effets en Argentine Contribution à une théorie de la politique linguistique. Thèse de doctorat EHESS. 1 vol.

\section{AUTEUR}

\section{ALICE BURROWS}

Diltec, Sorbonne Nouvelle-Paris 